\title{
Atom Probe Tomography of Zircon and Baddeleyite Geochronology Standards
}

\author{
D.A. Reinhard ${ }^{1}$, D.E. Moser ${ }^{2}$, I.R. Barker ${ }^{2}$, D. Olson ${ }^{1}$, I. Martin ${ }^{1}$, Katherine P. Rice ${ }^{1}$, Y. Chen ${ }^{1}$, D. \\ Lawrence $^{1}$, T.J. Prosa ${ }^{1}$ and D.J. Larson ${ }^{1}$ \\ 1. CAMECA Instruments Inc., Madison, WI USA \\ 2. University of Western Ontario, London, Ontario, CAN N6A 5B7
}

Atom probe tomography (APT) makes it possible to study the compositional structure of geological materials at the nanoscale [1]. It is an analytical technique whereby single atoms (or small groups of atoms) are ionized under the presence of a large electric field and removed from the surface one-at-atime. Each removal (field evaporation event) is synchronized to a timing pulse and projected to a 2D position-sensitive detector providing ion identification through time-of-flight mass spectroscopy and, ultimately, a composition map of the surface [2]. Performed iteratively over many millions of ions, the evolving surface information is reconstructed as a 3D map of individual ions from which information can be extracted for a for a variety of real-space analyses.

In general, optimal APT acquisition conditions are material dependent, thus experimentation is often necessary to achieve acceptable levels of data quality and analysis yield for an unfamiliar sample. As APT is a destructive technique, it may be advantageous to perform optimization on non-precious, standard materials prior to sacrificing the true material of interest. In the present work, we explore the effect of various laser-pulse energies and annealing conditions on zircon (BR266) [3] and baddeleyite (Phalaborwa) [4] standards and report progress to date.

Multiple APT specimens of both standards were made using standard lift-out techniques [2]. For many of the successful analyses, post-acquisition high-resolution SEM imaging was used to describe final specimen shape and constrain the reconstructed 3D ion maps (see Figure 1) [2]. Measurements of the final specimen apex radius, as a function of voltage and laser pulse energy, provides an estimate of the average electric field at the time of evaporation. Over the range of attempted pulse energies $(50-800 \mathrm{pJ})$, the evaporation field of zircon was observed to change by $12 \%$. Using a default k-factor of 3.3 [2], estimated evaporation fields ranged from $\sim 25-28 \mathrm{~V} / \mathrm{nm}$. Baddeleyite was found to behave similarly.

Because limited analysis yield is an issue [5], we explored post-specimen preparation treatments in hopes of improving the structural integrity. Annealing conditions ranging from $100-500{ }^{\circ} \mathrm{C}$ for $1-4$ hours are believed gentle enough to leave the primary structures unchanged while relaxing some of the internal stresses which might lead to premature specimen failure. Figure 2 shows atom maps from analyzed annealed and as-received specimens. No difference is observed in the size and distribution of detected $\mathrm{Pb}$-clusters between the annealed and un-annealed specimens.

\section{References}

[1] J.W. Valley et al., Nature Geoscience 7 (2014), p. 219.

[2] D.J. Larson et al., "Local Electrode Atom Probe Tomography" (Springer, New York 2014).

[3] R.A. Stern, Report 14, Geological Survey of Canada, Current Research, 2001-F.

[4] L.M. Heaman, Chemical Geology 261 (2009), p. 43.

[5] D.J. Larson et al., Microscopy and Microanalysis 20(S2) (2014), p. 2088. 

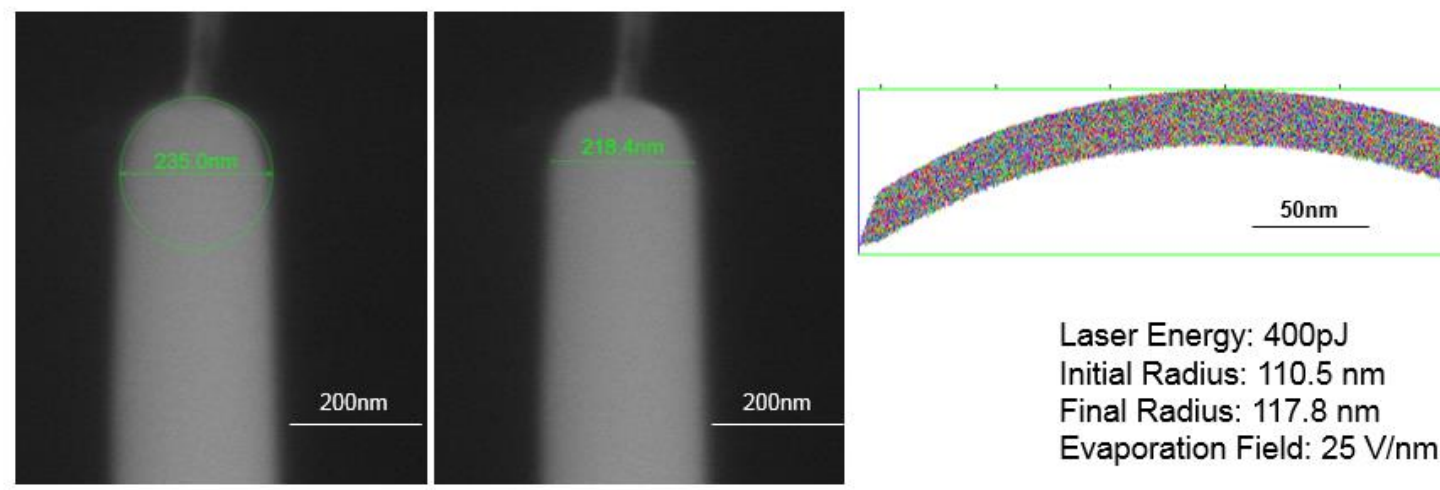

Figure 1: High-resolution SEM imaging of a zircon specimen after acquisition. These images were used to estimate the final tip radius, the shank angle and the sphere-to-cone ratio to constrain the reconstruction to allow for an estimate of the evaporation field for future acquisitions. Note: charging of the insulator specimen can cause aberrations in the image and limit the accuracy of the radius measurement.

$250^{\circ} \mathrm{C}$ anneal

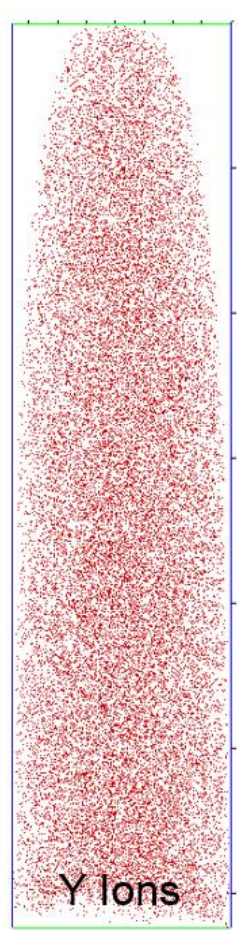

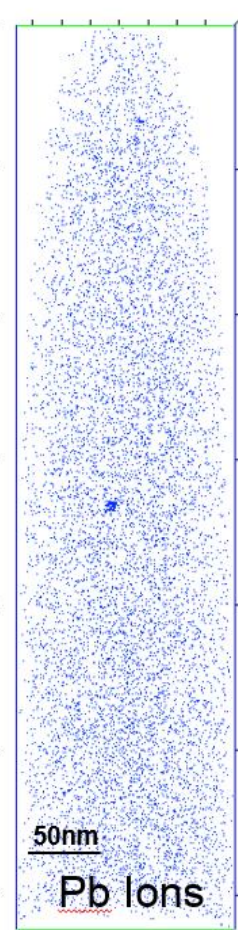

No anneal

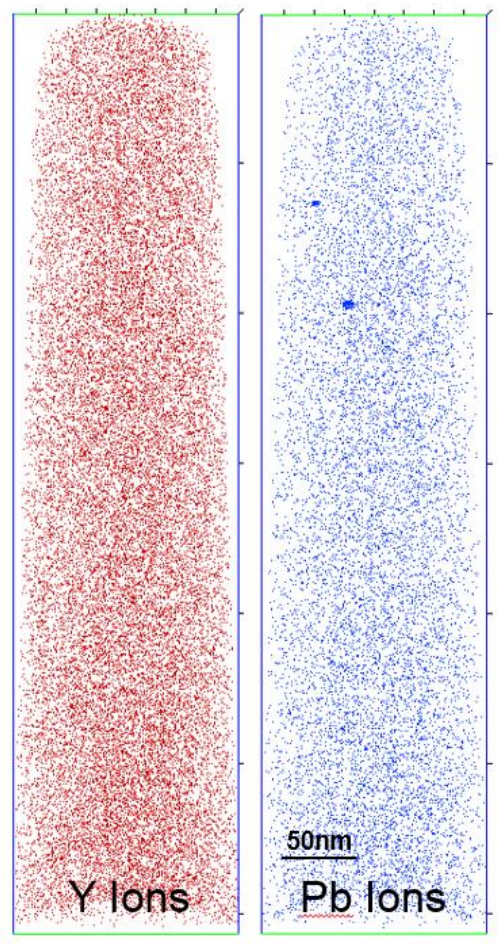

Figure 2: $\mathrm{Y}$ and $\mathrm{Pb}$ ion maps from annealed and un-annealed specimens showing similar spatial features. 